\title{
Enseñanza de idiomas mediante la acción social
} Language Training Through University Extension

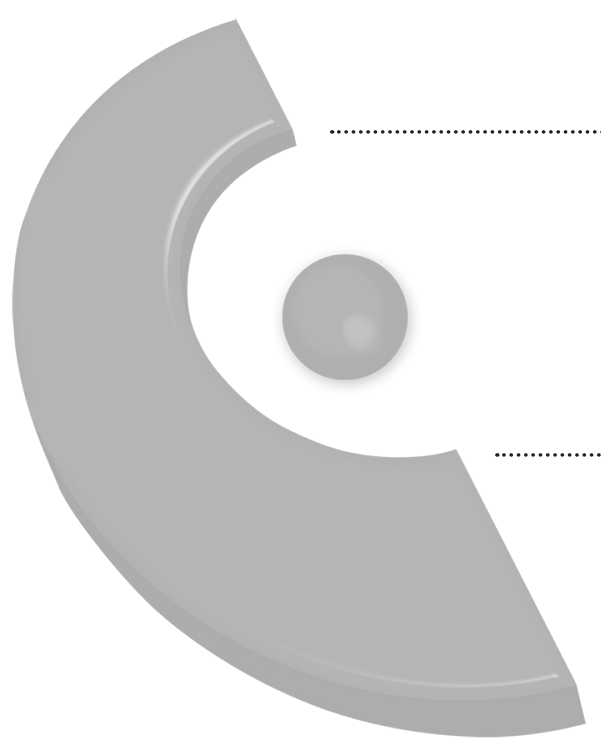

\author{
Rosberly López Montero ${ }^{l}$ \\ Universidad de Costa Rica \\ Sede del Pacífico \\ Puntarenas, Costa Rica \\ ros_ber_ly@yahoo.es
}

Recibido 26/10/2017 Aceptado 16/01/2018

Resumen. El presente artículo tiene como objetivo dar a conocer parte del trabajo realizado mediante el proyecto de Extensión Docente de la Vicerrectoría de Acción Social denominado Idiomas para la Comunicación Internacional, el cual se desarrolló en la Sede Regional del Pacífico de la Universidad de Costa Rica. Parte de este trabajo fue divulgado en el Taller de Extensión Universitaria del Congreso Internacional Universidad 2016 en la modalidad de póster. La importancia de aprender nuevos idiomas y facilitar posibilidades para su aprendizaje en las zonas fuera de la ciudad capital es una tesis que se defiende a lo largo de estas páginas y que se alcanza gracias a la acción social.

Palabras clave: extensión universitaria, idiomas, multilingüismo.

\footnotetext{
Máster en Lingüística de la Universidad de Costa Rica. Licenciada en la Enseñanza del Inglés. Docente e investigadora de la Sede del Pacífico de la Universidad de Costa Rica. Coordinadora del proyecto de Extensión Docente ED-2884: Idiomas para la Comunicación Internacional.
} 
Revista Universidad en DiÁlogo • Vol. 8, N. ${ }^{\circ}$ 1, Enero-Junio, 2018, pp. 11-22

ISSN 2215-2849 • EISSN: 2215-4752

DOI: http://dx.doi.org/10.15359/udre.8-1.1

\begin{abstract}
This paper aims to describe some of the work developed through using the Idiomas para la comunicación internacional (Languages for International Communication) Project, a university extension project the Pacific Regional Campus of University of Costa Rica has been conducting. Part of this paper was presented in the form of a poster in the Universidad 2016 International Congress. The importance of learning new languages and facilitating possibilities for their learning in regions outside the capital city is a thesis defended throughout these pages and is a goal reached thanks to university extension programs.
\end{abstract}

Keywords: languages, multilingualism, university extension.

\title{
Introducción
}

Actualmente, el conocimiento de otros idiomas es imperante ante las nuevas demandas laborales globales. Desafortunadamente, muchas de las oportunidades educativas en diversos campos, incluida la educación bilingüe, se enfocan en las áreas urbanas y centrales de los países, dejando de lado las zonas rurales o periféricas.

Las zonas rurales de Costa Rica, así como las provincias lejos de la ciudad capital, presentan una falta de acceso a servicios de educación vinculados a la instrucción de diferentes idiomas. Con respecto a esto, Acuña, Barahona y Ceciliano manifiestan que "la oferta pública debe considerar adecuados niveles de descentralización de los servicios educativos en idiomas, que ofrezcan el aprendizaje con criterios de calidad y eficiencia como se ofrecen en las regiones centrales del país" (2010, p. 17).

Esta situación es de particular importancia en un país cuya economía se encuentra altamente impulsada por el turismo internacional, siendo esta actividad una de las que más fuentes de trabajo genera a los habitantes de estas regiones. En este sentido, desde las sedes regionales de la Universidad de Costa Rica se tejen proyectos con proyección a la comunidad para fortalecer estas regiones y disminuir la brecha social y la disparidad existente entre comunidades periféricas y la ciudad capital. Específicamente en el área de idiomas, la Sede del Pacífico trabaja desde diciembre del 2011 para facilitar a las comunidades vecinas una opción académica permanente en capacitación de idiomas mediante el proyecto de extensión docente Idiomas para la Comunicación Internacional (ICI), por lo que en 
las siguientes líneas se dan a conocer los alcances de este proyecto en la región para facilitar la instrucción de idiomas y de esta manera contribuir a la reducción de la brecha social.

\section{Antecedentes}

\section{Del contexto sociogeográfico}

La Sede del Pacífico de la Universidad de Costa Rica, Arnoldo Ferreto Segura, se encuentra situada en el cantón Central de la provincia de Puntarenas. Esta sede universitaria ha tenido presencia en la región desde la década de los años setenta, pero es hasta el año 1992 que "el Consejo Universitario otorga el grado de Sede Regional del Pacífico a este centro de estudios superiores" (Chen, 2003, p. 8).

Según los datos del Instituto Nacional de Estadística y Censos (INEC, Censo 2011), este cantón tiene una población de 115019 habitantes, siendo el más habitado de toda la provincia de Puntarenas.

Además, de acuerdo con Alvarado y López (2012) esta provincia posee altos niveles de pobreza debido a diversos factores que han provocado un aumento en los niveles de desempleo y deserción escolar. Por consiguiente, la existencia de proyectos que permitan una activación de la economía y un progreso académico en la población es fundamental.

En esto contribuye la acción social, la actividad académica sustantiva de la Universidad de Costa Rica mediante la cual "se establece el vínculo Universidad-Sociedad de una manera dialógica y transitiva. Por ello, la Acción Social procura solidariamente, contribuir al desarrollo de capacidades en las comunidades con las que se relaciona" (Vicerrectoría de Acción Social, 2015), y parte de ello es el proyecto de Idiomas para la Comunicación Internacional, proyecto de acción social de la Sede Regional del Pacífico de la Universidad de Costa Rica.

\section{Del proyecto}

El proyecto Idiomas para la Comunicación Internacional ofrece capacitación a miembros de la comunidad en general mediante la modalidad de cursos libres. Su objetivo principal es promover el aprendizaje de idiomas 
Revista Universidad en DiÁlogo • Vol. 8, N. ${ }^{\circ}$ 1, Enero-Junio, 2018, pp. 11-22

ISSN 2215-2849 • EISSN: 2215-4752

DOI: http://dx.doi.org/10.15359/udre.8-1.1

en la comunidad de la provincia de Puntarenas, situada en el Pacífico del país, para que sus pobladores sean capaces de comunicarse con usuarios de diversas lenguas como un medio de apoyo a la diversidad lingüística y reducción de la brecha social.

La enseñanza de los cursos se realiza mediante el enfoque comunicativo y el aprendizaje colaborativo con una metodología interactiva que toma en cuenta los diferentes estilos de aprendizaje para satisfacer las necesidades de los estudiantes.

El proyecto está constituido por tres programas: Inglés para Jóvenes y Adultos, Inglés para Niños y Otros Idiomas. Este último constituye la parte más innovadora del proyecto, al no existir, al momento de su inicio, otro programa en la zona geográfica de impacto que ofreciera cursos libres de manera continua en idiomas aparte del inglés. Este programa ofrece cursos de Italiano, Portugués y Francés; adicionalmente, ha impartido cursos de Mandarín y de Lenguaje de Señas Costarricense (LESCO). La apertura de cursos en estos idiomas depende de la disponibilidad de docentes y de la población matriculada, lo cual, a su vez, representa uno de los retos más importantes de este proyecto, ya que en ocasiones se ha contado con docentes dispuestos a trasladarse a impartir estos cursos en la región, pero no hay suficiente población estudiantil, o viceversa. Sin embargo, como meta de este proyecto se estableció desde un inicio impartir cada período al menos un curso de un idioma que no sea inglés, meta que se ha cumplido hasta el día de hoy.

Mención aparte merece el programa Inglés para Niños, denominado oficialmente English for Kids. Este programa comienza un año después de iniciado el proyecto. La existencia de un nuevo programa de inglés para niños ha alcanzado una población mayor, ya que muchos padres y madres son conscientes de la importancia de aprender inglés desde pequeños, por lo que el programa English for Kids ha tenido un gran auge desde sus inicios, manejando una población de alrededor de cuarenta niños por período, de edades entre los tres y los doce años de edad. Al respecto, López y Rodríguez (2017) comentan que "su acogida en la comunidad ha sido perceptible por medio de la respuesta de la población y los bajos índices de deserción estudiantil” (p. 3).

Todos los cursos del proyecto son impartidos a lo largo de cuatro períodos al año, de diez semanas cada uno. El programa de cursos completo consta 
de un año y medio aproximadamente, realizándose un total de seis cursos; sin embargo, hay programas que han surgido como continuidad de estos, logrando que algunos programas tengan una duración de dos años o más, lo cual depende de cada población; lo más común es que esto suceda en las poblaciones más jóvenes, como niños o adolescentes.

En el caso del programa Inglés para Jóvenes y Adultos, el mismo se encuentra en una transición, pues el programa original constaba de seis módulos de sesenta horas cada uno; sin embargo, debido a lo observado en la población participante, se ha desarrollado un nuevo programa que se encuentra por iniciar, el cual consta de cinco módulos: los tres primeros de setenta horas y los dos últimos de treinta horas. Esto con el objetivo de lograr que una mayor cantidad de personas logre concluir el programa completo sin perder la calidad de este y así reducir de alguna manera un factor tan común en este tipo de cursos: la deserción, más notoria aún en las poblaciones adultas, debido a los diversos compromisos que enfrentan.

Asimismo, el programa Otros Idiomas tiene una duración de seis módulos de cuarenta horas cada uno, una sesión por semana y alberga la enseñanza de cualquier idioma que no sea inglés, para jóvenes y adultos. Por otro lado, el programa Inglés para Niños se divide en Inglés para Preescolares e Inglés para Escolares y consta de veinte horas por curso, son participativos, pues lo importante es que el estudiante aprenda en un ambiente libre de presión por sacar una determinada calificación, a la vez que desarrollan actitudes positivas hacia la lengua meta.

En el caso de Inglés para Escolares, una vez finalizado, se les da la opción de continuar con un programa de Talleres de Inglés Conversacional para Preadolescentes, de treinta horas por módulo para un total de cuatro módulos, y posterior al mismo, se inicia el programa de Talleres para el Fortalecimiento de Habilidades Específicas, los cuales les brindan herramientas para fortalecer el idioma en aspectos como la pronunciación, en destrezas de habla en público, comprensión auditiva y de lectura.

Vale mencionar que este es un proyecto autofinanciado, pero el precio establecido es más bajo de lo que usualmente se cobra por este tipo de cursos en otras zonas del país como la ciudad capital, por lo que se realizan grandes esfuerzos para mantenerlo sin elevar mucho los precios y con la intención de expandirlo a otras comunidades a costos aún más accesibles. 
Revista Universidad en DiÁlogo • Vol. 8, N. ${ }^{\circ}$ 1, Enero-Junio, 2018, pp. 11-22

ISSN 2215-2849 • EISSN: 2215-4752

DOI: http://dx.doi.org/10.15359/udre.8-1.1

\section{Desarrollo del tema}

\section{La importancia del multilingüismo}

Kemp (2009) manifiesta que los individuos multilingües pueden poseer niveles de competencia distintos de los idiomas que manejan, aunque no necesariamente deben manejar el mismo grado de competencia lingüística en estos idiomas.

En esta misma línea, López (2014) apunta que

Desde esta perspectiva, una provincia o región puede considerarse multilingüe si sus habitantes tienen algún conocimiento de otros idiomas, aunque el nivel de conocimiento o de competencia lingüística, en cada uno de esos idiomas, varíe. También, podría considerarse una región multilingüe, si los idiomas que manejan son distintos unos de otros (algunos saben italiano, otros francés o inglés) aunque el idioma oficial continúe siendo el español (p. 133).

Por lo anterior, ICI pretende contribuir al desarrollo del multilingüismo en la región, aspecto de alta importancia en la sociedad actual y del que ninguna comunidad debería quedar exenta. Al respecto, Rojas y Mauro (2012) manifiestan que "fomentar el aprendizaje de otros idiomas en la población representa el logro de metas políticas para una mejor inserción en un mundo globalizado" (p. 115). Esto indica la seriedad con la que debe tomarse el aprendizaje de nuevas lenguas en la creación de políticas educativas de los Gobiernos, ya que permitirá, entre otras cosas, que los habitantes compitan en el mercado global, creando mayores y mejores oportunidades para estos.

Así también, de acuerdo con un informe de la UNESCO del 2009, citado por los autores Rojas y Mauro (2012), los programas de educación bilingüe son fundamentales para mejorar la calidad educativa de aquellos con menores oportunidades económicas o sociales, es decir, la educación bilingüe es un motor para promover la equidad social, por lo que se retoma la idea de que las políticas educativas de una nación deben incluir el aprendizaje de idiomas extranjeros.

Por otra parte, el conocer otros idiomas atrae otros beneficios. Vásquez (2009) apunta que las personas que manejan al menos dos idiomas tendrán 
un impacto significativo en su desarrollo cognitivo, por lo que aquellos con acceso a programas bilingües tendrán mayores posibilidades de sobresalir en el ámbito académico.

\section{Impacto del proyecto.}

El impacto de este proyecto consiste en proporcionar el acceso al aprendizaje de otras lenguas, facilitando la incorporación a la comunidad internacional. Este impacto se ve reflejado en la cantidad de personas que responden al mismo, así como a la cantidad de cursos impartidos, lo que a su vez responde a las demandas nacionales e internacionales sobre la necesidad de conocer otro idioma, principalmente el inglés. Por otro lado, ha tenido un impacto a nivel académico, ya que permite el desarrollo de investigaciones en el ámbito de la lengua, de las cuales algunas ya han sido expuestas a la comunidad universitaria.

Por ejemplo, las clases de Inglés han brindado insumos para el desarrollo de investigaciones, lo que a su vez favorece el mejoramiento de los cursos. Una de estas investigaciones se basa en la discusión de grupos focales para explorar las razones por las que los participantes deciden tomar estos cursos, lo que de alguna manera proporciona detalles sobre el impacto que tiene el proyecto en la región y la consciencia que tiene la población de capacitarse en idiomas.

Entre estas razones se encuentran la posibilidad de un mayor desarrollo en lo referente a aspectos socioeconómicos, como el "acceso a mejores y más amplias oportunidades laborales" (López y Navarrete, 2013, p. 5), además, en el ámbito educativo los participantes de los cursos comentan que el tomar cursos de Inglés les facilita el acceso a becas y pasantías en el extranjero, así como alcanzar una meta personal; también, les brinda la posibilidad de viajar a otros países y conocer otros lugares y culturas (López y Navarrete, 2013).

En el caso específico del programa para niños, se han explorado las creencias de los estudiantes en etapa escolar con respecto a la lengua que estudian, lo cual ha demostrado resultados satisfactorios al indicar que la gran mayoría siente empatía por la lengua meta, así como por su docente y las clases como tal; dos individuos en dicho análisis indicaron desagrado y se logró determinar que era porque sentían dificultad al comprender el idioma (López, 2016), lo cual permite ejecutar acciones de mejora. 
Específicamente, estos cursos para niños inician desde la edad de los tres años, lo cual trae aún más beneficios a esta población, ya que tienen mayores posibilidades de aprender con éxito la lengua extranjera. Al respecto Brown (2007) comenta que los niños y las niñas que aprenden dos lenguas simultáneamente las adquieren a través de estrategias similares, pues se encuentran, en esencia, aprendiendo dos lenguas maternas, lo que hace el aprendizaje de lenguas extranjeras desde una edad temprana lo ideal.

En general, por período lectivo, la población estudiantil del proyecto es de un promedio de 130 estudiantes; de hecho, desde el año 2014 la población de estos cursos no ha sido menor de 100, algo que variaba en las estadísticas de los años anteriores, lo que demuestra la sostenibilidad del proyecto y la manera en que va teniendo un impacto en la región al reunir una mayor cantidad de estudiantes.

Seguidamente, se detalla cuántos cursos de inglés se han impartido tanto para niños como para adolescentes y adultos, y la cantidad de cursos impartidos en otros idiomas.

Cursos impartidos hasta el 2017

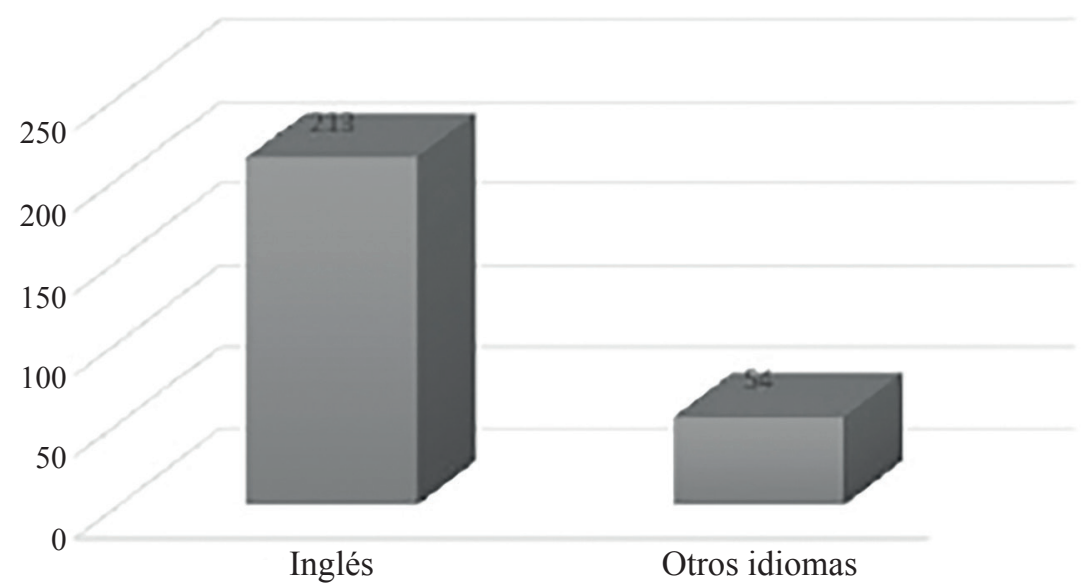

Figura 1. Cursos impartidos. Elaboración propia con base en registros del proyecto.

En el gráfico se observa que el idioma de mayor demanda es el inglés, 213 cursos en contraposición a 54 cursos de Italiano, Portugués, Francés, 
Mandarín y LESCO, lo que obedece a razones laborales y académicas; además, tiene mucho que ver con la apertura del programa de Inglés para Niños, que siempre ha tenido una matrícula alta.

Con respecto a los cursos en otros idiomas, el que más se ha impartido es el de Francés, seguido del curso de Portugués, como puede apreciarse abajo:

Otros idiomas impartidos

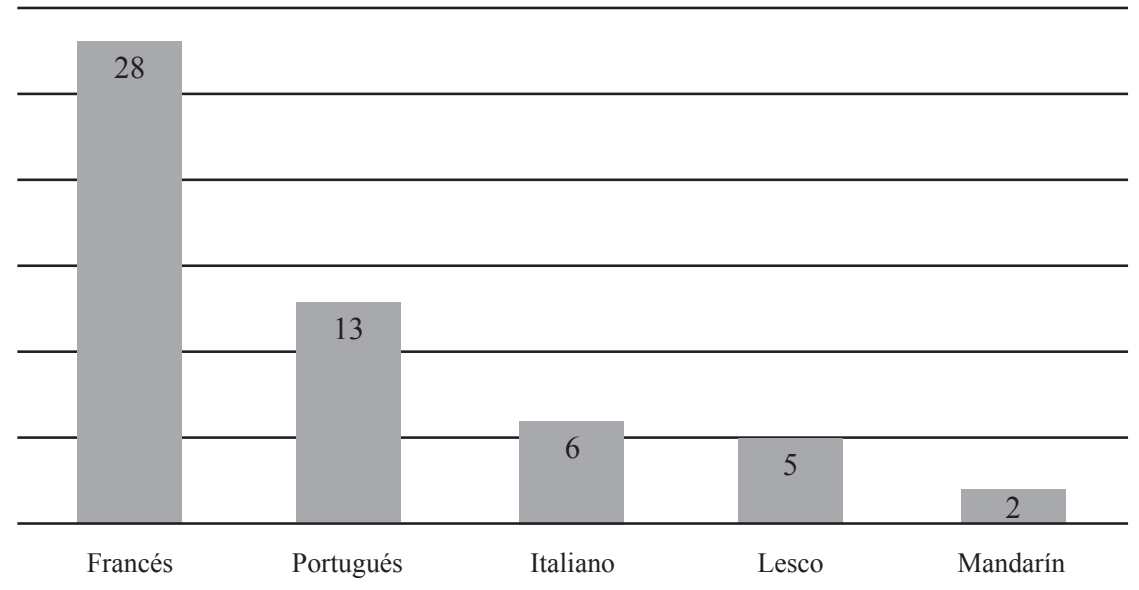

Figura 2. Otros idiomas impartidos. Elaboración propia con base en registros del proyecto.

Entre las razones por las que los estudiantes deciden estudiar otros idiomas se encuentra mayoritariamente el turismo, ya que algunos de ellos se desenvuelven en la industria; así también, placer, mayor competitividad a nivel laboral y afinidad con la cultura meta.

Aunque estos son los idiomas que se han impartido hasta el momento, no significa que sean los únicos que pueden llegar a impartirse, ya que el proyecto cuenta con la capacidad de crear nuevos programas en distintos idiomas con la colaboración de quienes se encuentren dispuestos a impartirlos, por supuesto, mientras la población lo desee; aspectos que también resultan ser causantes de que algunos idiomas se hayan ofrecido menos que otros, pero que no impide que puedan llegar a retomarse. 
Revista Universidad en DiÁlogo • Vol. 8, N. ${ }^{\circ}$ 1, Enero-Junio, 2018, pp. 11-22

ISSN 2215-2849 • EISSN: 2215-4752

DOI: http://dx.doi.org/10.15359/udre.8-1.1

\section{Conclusiones}

Vale destacar que este es un proyecto innovador en la zona en la que se encuentra. El mismo ha contribuido a la difusión del aprendizaje de otros idiomas en la comunidad a través de una educación permanente, consolidando el lazo universidad-sociedad. De esta manera, no es necesario para los pobladores trasladarse hasta la ciudad capital para aprender otros idiomas aparte del inglés, facilitando a las personas acceder a nuevas opciones de formación educativa, lo que fomenta el desarrollo local.

Con respecto a las limitaciones, en ocasiones se ofrece un curso, pero debe cerrarse por matrícula insuficiente, esto sucede mayoritariamente en cursos de otros idiomas; no obstante, desde el seno del proyecto se considera relevante mantener una oferta de otros idiomas para contribuir en el aprendizaje de otras lenguas y favorecer el multilingüismo en la región, lo importante es mantener abierta la posibilidad.

Además, el proyecto cuenta con un programa de francés para niños, el cual no ha sido posible iniciar debido a que padres y madres de familia suelen preferir inscribir a sus hijos en cursos de Inglés antes que en otro idioma; sin embargo, se seguirá intentando ofrecer nuevos idiomas a la población infantil, lo cual representa uno de los mayores retos.

Aunado a esto, se trabaja para impartir cursos en otras comunidades aún más lejanas de la ciudad capital, a precios acordes con la realidad socioeconómica de cada zona; de modo similar, se pretende fortalecer un programa de reducción de costos para estudiantes del sistema educativo del país con limitaciones económicas y de este modo propiciar, en la medida de lo posible, mayores opciones para los habitantes de las comunidades puntarenenses.

Lo que es valioso destacar es el camino de posibilidades que se va forjando a través de los años en las comunidades mediante la acción social. Específicamente, las sedes regionales de la Universidad de Costa Rica cumplen una gran labor a través del desarrollo de proyectos de extensión docente, extensión cultural y trabajo comunal universitario, en este caso específico en la proyección y capacitación de idiomas. 


\section{Referencias}

Acuña, G., Barahona, M. y Ceciliano, Y. (2010). Extensión y dominio efectivo del inglés como segunda lengua en el sistema educativo costarricense: situación actual, problemas y desafios. III Informe del Estado de la Educación. CONARE.

Alvarado, L. y López, R. (2012). El nivel de escolaridad y actitudes lingüísticas de los puntarenenses. Ponencia presentada en las III Jornadas de Investigación del Pacífico Costarricense, Puntarenas, Costa Rica.

Brown, D. (2007). Principles of Language Learning. (3 ${ }^{\text {a }}$. ed.). Nueva York: Pearson Education.

Chen, S. (2003). Presentación. Revista Inter Sedes, 4(7), 7-9.

Instituto Nacional de Estadística y Censos (2011). Censo. Recuperado de http://www.inec.go.cr/Web/Home/GeneradorPagina.aspx

Kemp, C. (2009). Defining multilingualism. Exploration of Multilingualism: Development on Research on L3, Multilingualism and Multiple Language Acquisition. Amsterdam: John Benjamin Publishing Company.

López, R. (2014). Puntarenas hacia el camino del multilingüismo: impacto del Proyecto de Idiomas de la Sede del Pacífico de la Universidad de Costa Rica. Revista InterSedes, 15(30), 132-150.

López, R. (2016). Creencias sobre el aprendizaje del idioma inglés desde la perspectiva del estudiantado infantil. Revista Extensión en Red, (7), 100-115. Recuperado de http://perio.unlp.edu.ar/ojs/index.php/ extensionenred/article/view/3434/2937

López, R. y Navarrete, K. (2013). El inglés como herramienta de desarrollo en Puntarenas: algunas consideraciones motivacionales. Ponencia presentada en las IV Jornadas de Investigación del Pacífico Costarricense, Puntarenas, Costa Rica.

López, R. y Rodríguez, K. (2017). Reflexiones del programa de extensión docente English for Kids de la Sede del Pacífico de la Universidad de Costa Rica. Revista Educación, 41(1), 1-15. Recuperado de http://dx.doi.org/10.15517/revedu.v41i1.19285 
Revista Universidad en DiÁlogo • Vol. 8, N. ${ }^{\circ}$ 1, Enero-Junio, 2018, pp. 11-22

ISSN 2215-2849 • EISSN: 2215-4752

DOI: http://dx.doi.org/10.15359/udre.8-1.1

Rojas, R. y Mauro, S. (2012). Eficacia e impacto en la implementación del programa ED-93 Idiomas para la Comunicación Intercultural. Revista de Lenguas Modernas, (16), 113-128.

Vásquez, L. (2009). Cognitive advantages of balanced bilingualism. Revista Pensamiento Actual, 9(12-13), 69-78. Recuperado de https:// revistas.ucr.ac.cr/index.php/pensamiento-actual/article/view/2837

Vicerrectoría de Acción Social. (2015). ¿Qué es acción social? Recuperado de http://accionsocial.ucr.ac.cr/accion-social 